Thorax (1970), 25, 751.

\title{
Lung manifestations in leptospirosis
}

\author{
S. C. POH and C. S. SOH \\ Tan Tock Seng Hospital, Singapore
}

\begin{abstract}
Two cases of leptospirosis presenting with cough and haemoptysis are described, together with their pulmonary radiological features. The various radiological chest findings are reviewed together with the pathogenesis and pathology of the lesions. Although the pulmonary involvement due to a haemorrhagic pneumonitis in leptospirosis is usually an incidental finding, occasional.y it is the most pronounced clinical manifestation of the disease, and may present a difficult diagnostic problem. It has to be considered in the differential diagnosis of diffuse miliary infiltrations or consolidation seen on the chest radiographs, especially in countries where both pulmonary tuberculosis and leptospirosis are not uncommon diseases.
\end{abstract}

Leptospirosis is a common condition in tropical and subtropical countries. The typical clinical picture is characterized by fever, conjunctivitis, jaundice, muscle pains, and leucocytosis. In about $30 \%$ of patients jaundice is absent, and pulmonary, influenzal or meningeal symptoms may predominate. There have been few reports of the pulmonary manifestations in leptospirosis (Silverstein, 1953; Ch'iu and Liu, 1959; Wang, Chi, and $\mathrm{Lu}, 1965 \mathrm{~b})$. Haemoptysis due to haemorrhagic pneumonitis is occasionally an early and prominent clinical feature and may present the clinician with an unusual diagnostic problem. We report here two cases of leptospirosis, first presenting with haemoptysis and mistakenly diagnosed initially on the radiological findings as pulmonary tuberculosis.

\section{CASE REPORTS}

CASE 1 A 41-year-old Malay police constable was admitted to the Tan Tock Seng Hospital on 18 March 1969 complaining of fever and cough with bloodstained sputum of three days' duration. He had been well until five days before admission, when he developed a fever associated with chills and rigors. Two days later he began to have a hacking cough productive of blood-stained sputum. At the same time he began to experience some weakness of the legs. A chest radiograph on 17 March 1969 had been reported as 'showing extensive markings in all lung fields presumably due to pulmonary tuberculosis' (Fig. 1).

On examination the temperature was $103^{\circ} \mathrm{F}$., pulse 96/minute: he appeared toxic and acutely ill. The sclerae were icteric; the liver was enlarged $3 \mathrm{~cm}$. below the costal margin and crepitations were heard over the right lower lobe. Urine analysis showed a trace of bile and urobilin, some albuminuria and a few red blood cells. The haemoglobin was 10 g. $/ 100$ $\mathrm{ml}$. and the white count was $16.200 / \mathrm{c} . \mathrm{mm}$., with $89 \%$ polymorphonuclear leucocytes. The platelet count was $96,000 /$ c.mm. Sputum examination for acid-fast bacilli was negative. Serum electrolytes and blood urea were normal. The serum bilirubin was $12 \mathrm{mg}$. $/ 100 \mathrm{ml}$., alkaline phosphatase $21 \cdot 2 \mathrm{~K}$.A. units / $100 \mathrm{ml}$ and serum glutamic pyruvic transaminase was 149 K.A. units $/ 100 \mathrm{ml}$. The electrocardiogram was normal. The chest radiograph on the day of admission showed extensive patchy consolidation in both lung fields, appearing confluent in the right upper and mid zones (Fig. 2). The sensitized erythrocyte lysis test gave a positive titre of $1: 1,600$, rising later to $1: 6,400$.

He was treated as a case of leptospirosis with crystalline penicillin, 4 mega units intramuscularly daily. He remained moderately ill until the fourth day, when the temperature returned to normal. His haemoptysis ceased on the second day after admission. The opacities on the chest film resolved rapidly (Fig. 3) and by the 10th day of his admission had completely disappeared (Fig. 4).

CASE 2 A 21-year-old Chinese man was admitted to another hospital on 19 December 1969 with a history of fever and giddiness for five days. Clinical examination did not reveal any abnormality except for a temperature of $102^{\circ} \mathrm{F}$. On the fifth day of his admission, he coughed up some blood-stained sputum. A chest radiograph taken on the following day showed generalized nodular mottlings over both lung fields, particularly in the right lung (Fig. 5). In view of these findings he was transferred to the Tan Tock Seng Hospital as a possible case of miliary tuberculosis.

Physical examination on admission revealed a pale, febrile, toxic patient with mild icterus. A soft liver was palpable $1 \mathrm{~cm}$. below the right cestal margin. 


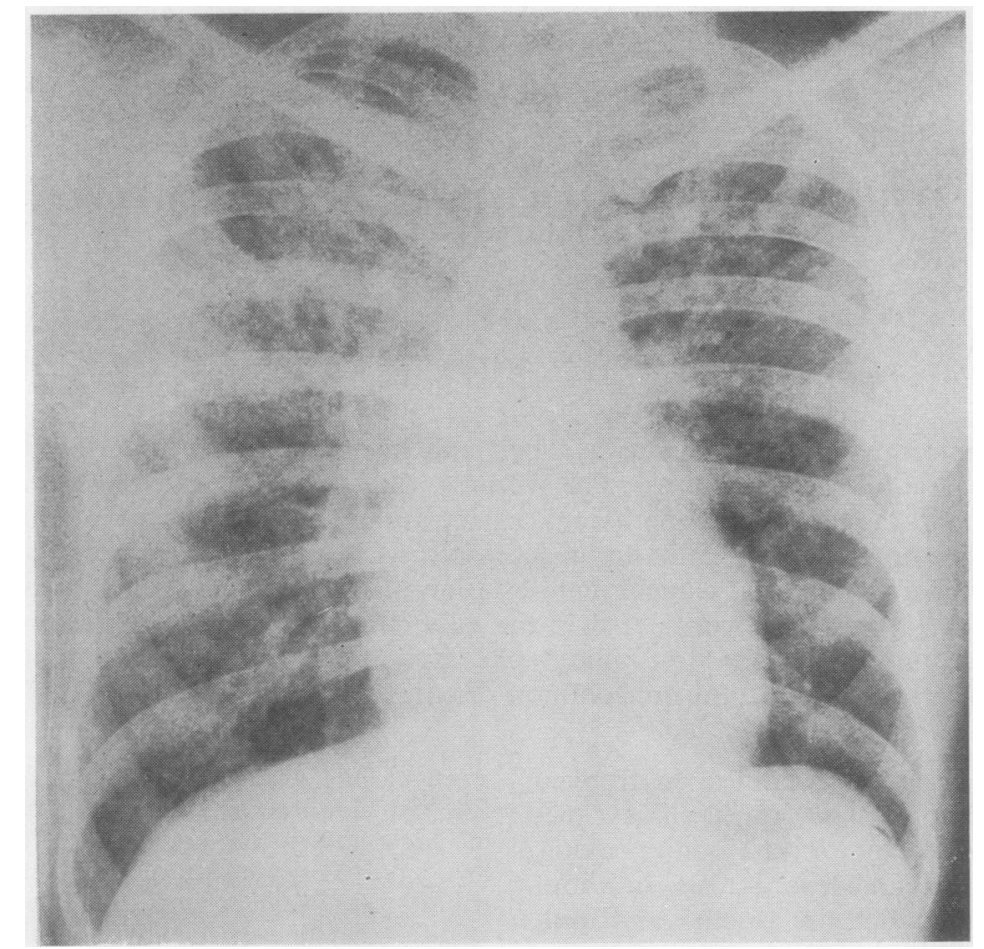

FIs. 1. Cas? 1. Chest radiograph on the day before admission shows extensive opacities in both lungs.

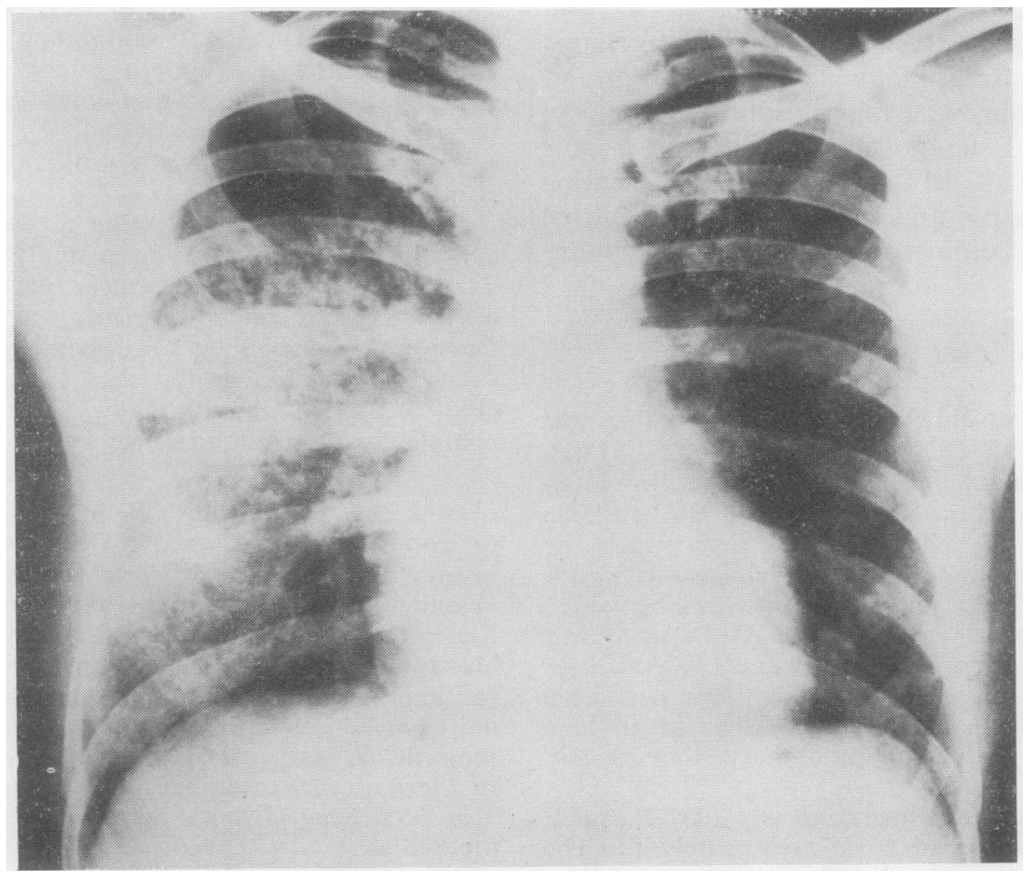

FIG. 2. Case 1. Chest radiograph on day of admission reveals opacities in both lungs with patchy consolidation on the right. 


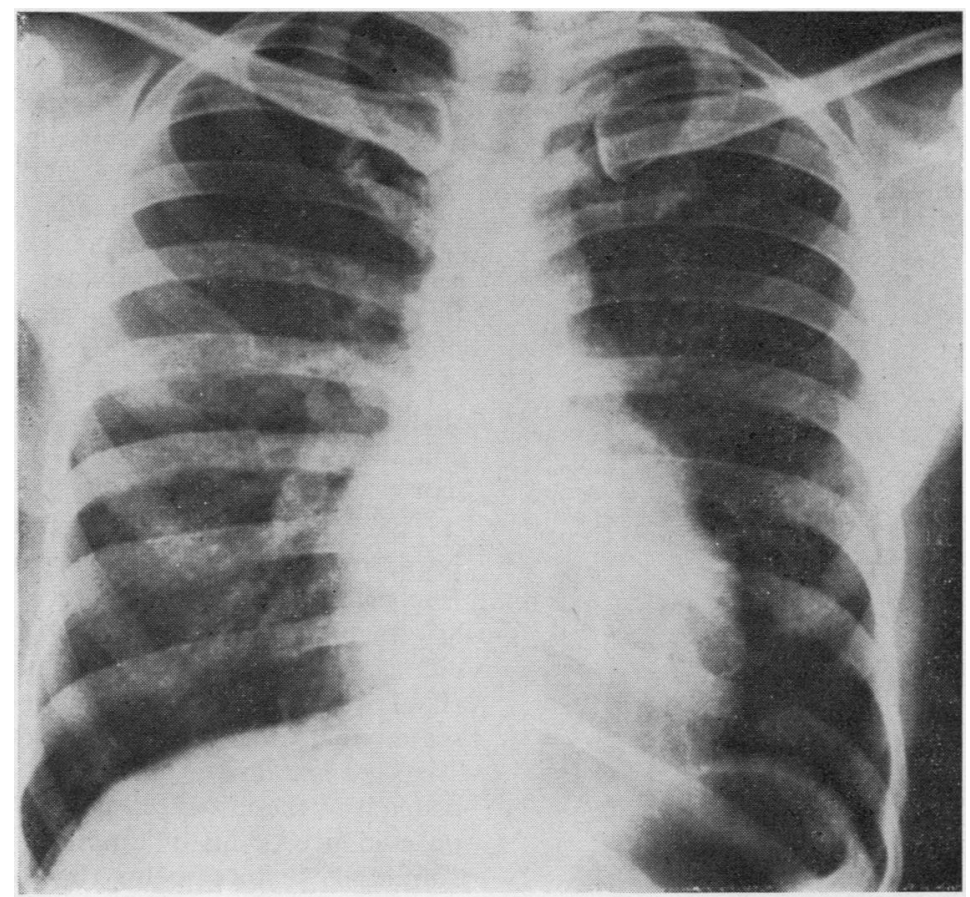

FIG. 3. Case 1. Chest radiograph three days after admission shows rapid resolution of the opacities.

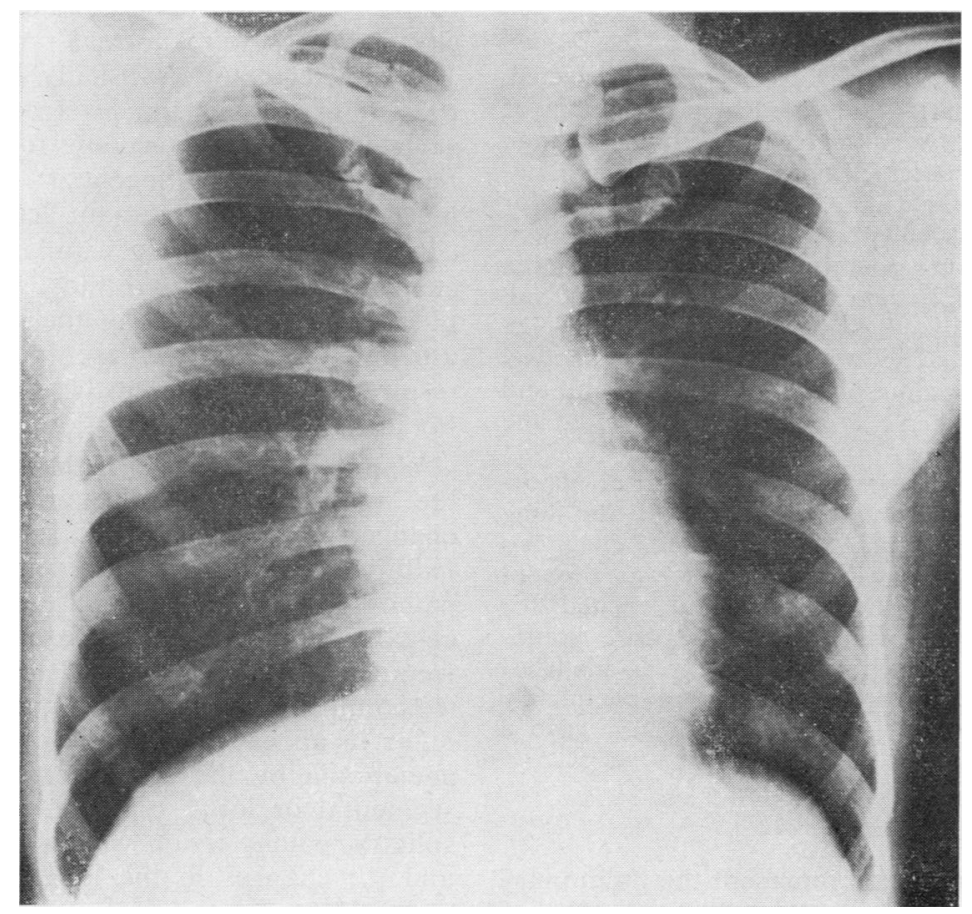

FIG. 4. Case 1. Normal radiograph 10 days after admission. 


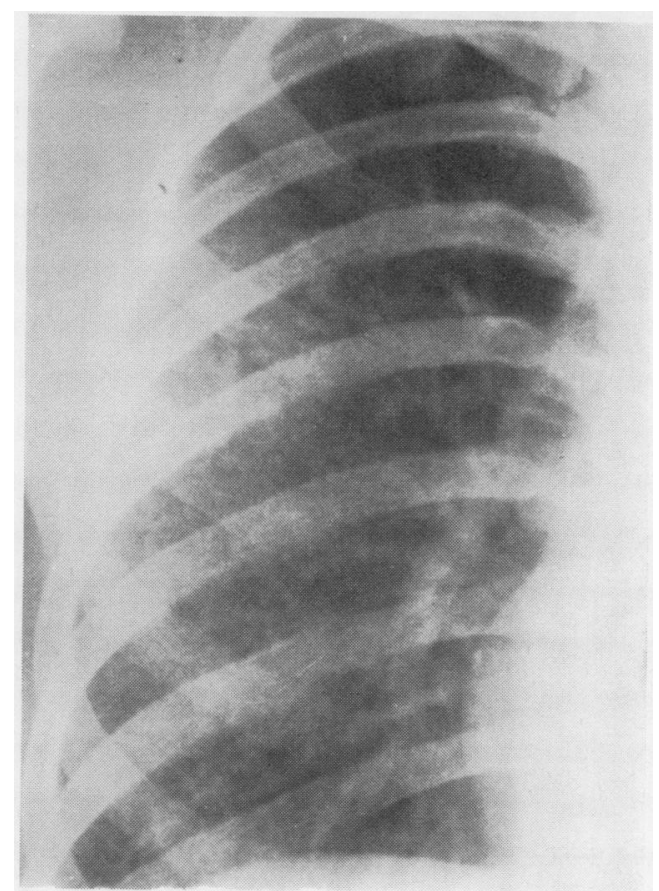

FIG. 5. Case 2. Chest radiograph on sixth day of illness reveals generalized nodular mottling, especially in the right lung.

No other abnormal physical signs were detected. Laboratory investigations revealed a haemoglobin of 7.9 g. $/ 100 \mathrm{ml}$., and a white count of 19,000/c.mm., with $78 \%$ polymorphonuclear leucocytes. The platelet count was $125,000 /$ c.mm. Sputum examination for acid-fast bacilli was negative. Urine analysis showed albuminuria and some granular casts. The blood urea was $59 \mathrm{mg}$. $/ 100 \mathrm{ml}$., serum bilirubin $3.0 \mathrm{mg} . / 100 \mathrm{ml}$. and the serum glutamic pyruvic transaminase 325 K.A. units $/ 100 \mathrm{ml}$. The cerebrospinal fluid showed a cell count of $44 /$ c.mm., protein $30 \mathrm{mg}$. $/ 100 \mathrm{ml}$. and sugar $52 \mathrm{mg} . / 100 \mathrm{ml}$. No organisms were seen on the smear.

A repeat chest radiograph on the day after admission showed almost complete clearing of the lung opacities. In view of this, the provisional diagnosis of miliary tuberculosis with meningitis was thought unlikely and he was investigated and treated for leptospirosis with penicillin. His temperature gradually settled and by the end of the first week his chest radiograph and cerebrospinal fluid were completely normal. The sensitized erythrocyte lysis test gave a positive titre of $1: 6.400$.

\section{DISCUSSION}

The two patients reported represent the pu'monary form of leptospirosis as described by Wang, Liu, Chang, Cheng, Luo, and Hung (1965a).
It is believed that the severity and clinical presentations of leptospirosis are not related to the various serotypes and that the clinical manifestation depends mainly on the virulence of the spirochaete involved as well as on individual reactivity. In the pulmonary form, cough and haemoptysis appear early in the course of the disease, usually within 72 hours following the abrupt febrile onset. The haemoptysis is usually scanty and clears within three to five days. Occasionally, massive haemoptyses might occur and cause asphyxiation.

The pathology of the pulmonary lesions has been reported in only a few cases (Havens, Bucher, and Reimann, 1941; Moeschlin, 1943; Silverstein, 1953; Cheng and Ch'eng, 1963). The changes described consist chiefly of a haemorrhagic pneumonitis. Petechiae and ecchymoses are scattered throughout both lungs and pleurae, with areas of extensive pulmonary haemorrhage and oedema in the advanced cases. Microscopy reveals no evidence of an inflammatory process, the predominant feature being haemorrhagic changes. Spirochaetes have been recovered from the lungs.

The haemorrhagic diathesis has been attributed to disturbances in liver function and in the clotting mechanism. However, the liver has been found to be little damaged and the bleeding and clotting times were normal (Wang et al., 1965a). The platelet count is usually lowered, as in our present cases, but the level appears to be of a degree inadequate to account for the haemorrhagic tendency. The pathogenesis of the lesions is believed to be due to toxin acting directly on the capillaries, resulting in capillary damage (Silverstein, 1953). Lesions are most marked in the peripheral and basal areas of the lung and have been attributed to the increase in capillarity and in respiratory excursion in these areas (Wang et al., 1965b).

Wang et al. (1965b), in their detailed report on the radiological manifestations of pulmonary changes in leptospirosis, described four different features consisting of (a) snowflake-like, small, patchy lesions, either restricted to a few intercostal spaces or widely disseminated, and more frequent in the peripheral parts of the lung; (b) confluent massive areas of consolidation which differ in appearance from classical pneumococcal pneumonia by the absence of sharply delineated segmental or lobar types of consolidation; $(c)$ a solitary, patchy lesion with an ill-defined margin ; and $(d)$ changes in the lung markings. The first type was by far the most common, being seen in $63 \%$ of their cases with positive radiographic 
findings. However, combinations of the various types were commonly present. No pleural effusion or hilar enlargement was noted. The radiographic picture of our first case shows a combination of small disseminated lesions with areas of consolidation, whereas that of the second case showed only the small disseminated opacities. Whilst the presence or absence of haemoptysis does not always indicate the extent of the lesions, usually patients with severe haemoptysis tend to show more extensive lesions. Resolution of the lesions, as shown in our two cases, is usually complete within 10 days, there being no sequelae left in the areas affected.

\section{REFERENCES}

Cheng, W. J., and Ch'eng. T. C. (1963). Pathologic findings on 5 cases with anicteric leptospirosis. Zhonghuo Neike Zazhi, 11, 208. [Chinese J. Internal Med.] Peking.

Ch'iu, Y. C., and Liu, H. H. (1959). Report on observation of roentgenologic pulmonary changes in 48 cases of leptospirosis. Zhonghuo Fangshexue Zazki, 7, 374. [Chinese J. Radiology.] Peking.

Havens, W. P., Bucher, C. J., and Reimann, H. A. (1941). Leptospirosis: a public heaith hazard. Report of a small outbreak of Weir's disease in bathers. J. Amer. med. Ass., 116, 289.

Moeschlin, S. (1943). Lungeninfiltrate beim Ikterus infectiosus Weil. Schweiz. med. Wschr., 73, 1227.

Silverstein, C. M. (1953). Pulmonary manifestations of leptospirosis. Radiology, 61, 327.

Wang, C. N., Liu. J., Chang, T. F., Cheng, W. J., Luo, M. Y., and Hung, A. T. (1965a). Studies on anicteric leptospirosis. I. Clinical manifestations and antibiotic therapy. Chin. med.J., 84, 283.

Wang, C. P., Chi, C. W., and Lu, F. L. (1965b). Studies on anicteric leptospirosis. III. Roentgenologic observations of pulmonary changes. Chin. med. J., 84, 298. 